\title{
Aneurisma chagásico do ventrículo esquerdo: aspectos cirúrgicos em 29 casos
}

José WANDERLEY NETO*, Luiz Daniel F. TORRES*, Decio O. ELIAS*, Sandra Tenório da SILVA*, Hemerson C. GAMA*, Mônica FARIAS*, Gilvan O. DOURADO*, Cid C. CAVALCANTE*, Antonio De Biase WISZOMIRSKY*, José Teles de MENDONÇA*.

WANDERLEY NETO, J.; TORRES, L. D. F.; ELIAS, D. O.; SILVA, S. T.; GAMA, H. C.; FARIAS, M.; DOURADO, G. O.; CAVALCANTE, C. C.; WISZOMIRSKY, A. D. B.; MENDONÇA, J. T. - Aneurisma chagásico de ventrículo esquerdo: aspectos cirúrgicos em 29 casos. Rev. Bras. Cir. Cardiovasc., 7(3): 180-185, 1992.

RESUMO: Entre março de 1980 e dezembro de 1991, 29 pacientes foram submetidos a remoção cirúrgica de aneurisma do ventrículo esquerdo de etiologia chagásica. Vinte e dois pacientes eram do sexo feminino e 7 do masculino, com idades que variaram entre 21 e 69 anos, todos com reação imunológica positiva para doença de Chagas. No pré-operatório, 8 pacientes apresentaram acidente vascular cerebral, 8 tinham cansaço (algum grau de insuficiência cardíaca congestiva), 8 tinham palpitaçōes e tonteiras, enquanto os 5 restantes apresentaram associaçōes dessas queixas. Três pacientes eram portadores de marcapasso artificial. $O$ eletrocardiograma estava alterado em todos os casos. A radiografia de tóraxera normal em metade dos casos. Do ponto de vista angiográfico, as coronárias eram normais e o ventrículo esquerdo apresentava aneurisma apical, com aspecto de dedo de luva ou mamilo em 14 pacientes, sacular em 8, de parede inferior em 4 e duplo (inferior + ponta) em 3. Em um dos pacientes (duplo aneurisma) havia insuficiência mitral importante e em 4 detectou-se a presença de trombos intracardíacos. Não houve mortalidade hospitalar. No seguimento pós-operatório, houve um óbito. Três pacientes desenvolveram bloqueio atrioventricular completo e necessitaram implante de marcapasso. Um paciente, cuja indicaçāo era arritmia, teve recorrência dos sintomas. Concluímos que a ressecção de aneurismas de etiologia chagásica, em casos selecionados, é procedimento de baixa mortalidade, previne a recorrência de tromboembolismo, controla a insuficiência cardíaca devido à discinesia e pode eliminar arritmias em um significativo número de pacientes.

DESCRITORES: aneurismas chagásicos, cirurgia; aneurismas de ventrículo esquerdo, cirurgia.

\section{INTRODUÇĀO}

O aneurisma de ventrículo de natureza chagásica é um achado freqüente. Foi descrito por Carlos Chagas, em $1916^{12}$, tem vasta sinonímia e é considerado lesão característica da doença de Chagas.

Em achados de necropsia o adelgaçamento da musculatura da ponta do ventrículo é encontrado em até $86 \%$ dos casos e pode se manifestar em outras paredes da cavidade ventricular esquerda e no ventrículo direito ${ }^{2,11,14}$.
Sua relação com morte súbita, arritmias e tromboembolismo é conhecida.

A possibilidade de tratamento cirúrgico em alguns casos foi discutida por alguns autores e executado por outros, sem, entretanto, ficarem estabelecidos 0 alcance $e$ a indicação precisa da cirurgia $6,8,9,16,17,23,27$.

O objetivo de nosso estudo é revisar o assunto e expor a nossa experiência com 29 casos operados, procurando contribuir para o melhor conhecimento da doença e sua indicação cirúrgica.

\footnotetext{
Trabalho realizado no Instituto de Doenças do Coração da Santa Casa de Misericórdia de Maceió. Maceió, AL, Brasil.

* Do Instituto de Doenças do Coraçáo.

Endereço para separatas: José Wanderley Neto. Rua Barão de Maceió, 288. Centro. 57000 Maceió, AL.
} 
WANDERLEYNETO, J.; TORRES, L. D. F.; ELIAS, D. O.; SILVA, S. T.; GAMA, H. C.; FARIAS, M.; DOURADO, G. O.; CAVALCANTE, C. C.; WISZOMIRSKY, A. D. B.; MENDONÇA, J. T. - Aneurisma chagásico de ventrículo esquerdo: aspectos cirúrgicos em 29 casos. Rev. Bras. Cir. Cardiovasc., 7(3): 180-185, 1992.

\section{CASUÍSTICA E MÉTODOS}

Foram estudados 29 pacientes portadores de aneurisma chagásico de ventrículo esquerdo, submetidos a tratamento cirúrgico, entre março de 1980 e dezembro de 1991.

As idades variaram de 21 a 69 anos. Vinte e dois pacientes eram do sexo feminino e 7 do masculino.

As manifestaçōes clínicas apresentadas pelos pacientes eram de acidente vascular cerebral em 8 , cansaço (algum grau de insuficiência cardíaca congestiva) em 8, tonteiras e palpitaçōes em 8 e 5 pacientes tinham queixas diversas. Três pacientes eram portadores de marcapasso.

O eletrocardiograma estava alterado em todos os casos, não se apresentando característico da lesão; os achados freqüentes foram bloqueio de ramo direito associado a hemibloqueio anterior esquerdo, bloqueio atrioventricular total e áreas inativas.

O ecocardiograma não foi realizado em todos os pacientes, porém, naqueles em que se utilizou o ecocardiograma bidimensional, a lesão foi demonstrada com facilidade (Figura 1).

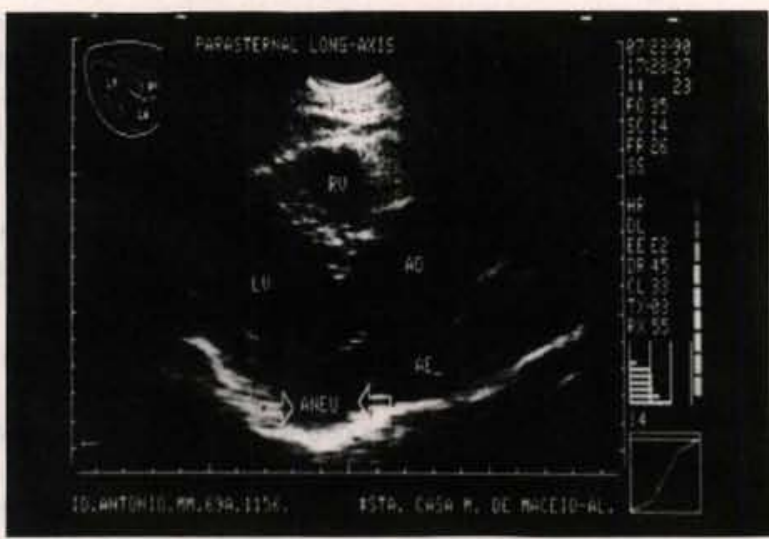

Fig. 1 - Aspecto ecocardiográfico mostrando o aneurisma apical destacado entre as setas.

A radiografia de tórax mostrou área cardíaca normal em metade dos casos; em $30 \%$ havia pequeno aumento da área cardíaca e em $20 \%$ o aumento era moderado. $O$ índice cardiotorácico variou de 0,45 a 0,60 .

Estudo hemodinâmico foi realizado em todos os casos e consistiu de cinecoronariografia, ventriculografia esquerda e medida das pressōes intracavitárias. As coronárias eram normais em todos os pacientes. A pressão distólica final do ventrículo esquerdo estava alterada em 6 casos, variando de 16 a $22 \mathrm{mmHg}$. A pressão pulmonar estava elevada $(60 \mathrm{mmHg})$ em apenas um paciente.
A ventriculografia esquerda evidenciou aneurisma apical com aspecto de de do de luva ou de mamilo em 14 pacientes. $O$ aspecto da lesão era sacular em 8 (Figura 2). Em 4 pacientes o aneurisma estava localizado na parede inferior do ventrículo esquerdo. Em 3 a lesāo era dupla, associada, de parede inferior e apical (Figura 3 ). Em 1 caso havia insuficiência mitral severa (Figura 4) e outros 4 pacientes tinham trombos no interior do aneurisma (Figura 5).

O tratamento cirúrgico foi realizado com circulação extracorpórea, hemodiluição e hipotermia leve ou normotermia.

A área aneurismática era incisada e o interior do ventrículo esquerdo examinado. As bordas da lesāo eram ressecadas e o ventrículo suturado com apoio de tiras de pericárdio bovino. Em 8 casos a plastia da ponta do ventrículo foi precedida de cerclagem do colo do aneurisma por sutura em bolsa (remodelagem geométrica), de acordo com os conceitos emitidos por JATENE ${ }^{15}$.

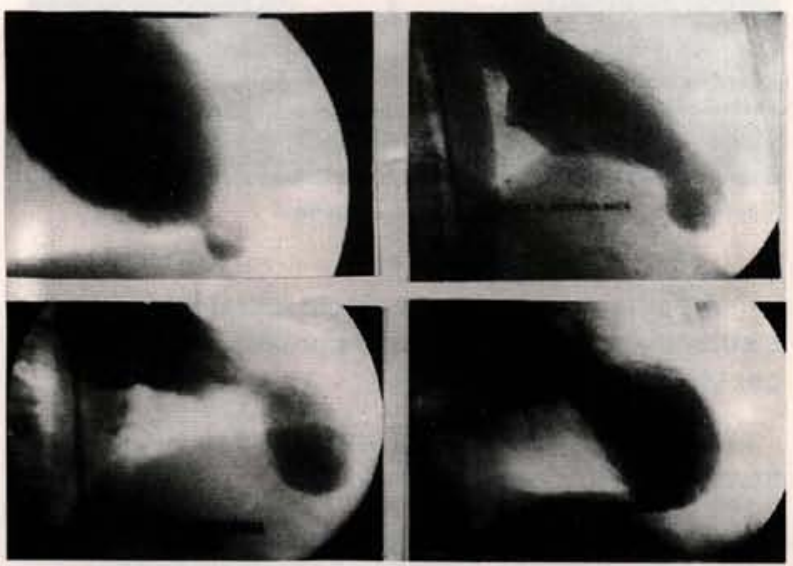

Fig. 2 - Aspecto angiográfico dos aneurismas mamilar, dedo de luva e sacular.

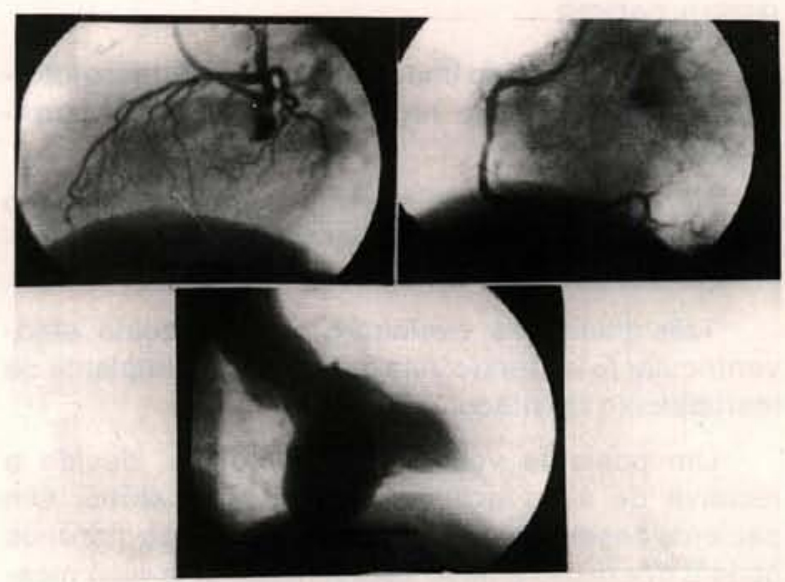

Fig. 3-Aneurisma duplo (apical e inferior) sem insuficiência mitral. 
WANDERLEY NETO, J.; TORRES, L. D. F.; ELIAS, D. O.; SILVA, S. T.; GAMA, H. C.; FARIAS, M.; DOURADO, G. O.; CAVALCANTE, C. C.; WISZOMIRSKY, A. D. B.; MENDONÇA, J. T. - Aneurisma chagásico de ventrículo esquerdo: aspectos cirúrgicos em 29 casos. Rev. Bras. Cir. Cardiovasc., 7(3): 180-185, 1992.

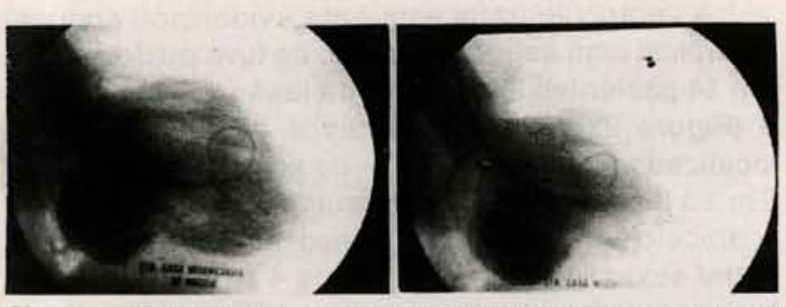

Fig. 4-Aneurisma apical e de parede inferior com insuficiência mitral grave.

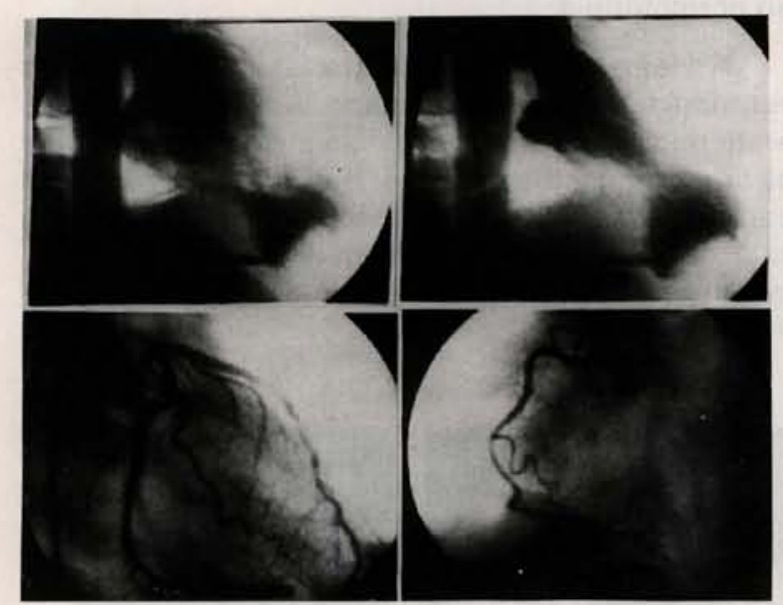

Fig. 5 - Aneurisma sacular com trombose.

A paciente com insuficiência mitral foi submetida a substituiçāo da valva mitral por bioprótese porcina, por via atrial esquerda.

Em 4 pacientes foram encontrados e removidos trombos no interior do aneurisma.

O exame histopatológico mostrou fibrose, com diferentes graus de infiltrado linfoplasmocitário.

\section{RESULTADOS}

O pós-operatório imediato transcorreu sem intercorrências dignas de registro e nāo houve mortalidade hospitalar.

No seguimento pós-operatório, houve um único óbito, seis meses após a operaçāo, por causa desconhecida.

Três pacientes evoluiram para bloqueio atrioventricular total, tendo sido submetidos a implante de marcapasso cardíaco.

Um paciente voltou a ter sintomas, devido a recidiva de suas arritmias no pós-operatório. Um paciente desenvolveu insuficiência cardiaca dez anos após a operaçāo e novo estudo hemodinâmico mostrou piora global da função ventricular esquerda.

\section{COMENTÁRIOS}

O aneurisma apical tem sido descrito com vasta sinonímia (adelgaçamento ventricular, endocardite parietal crônica, lesão necrótica apical, lesão vorticilar, lesão fibrótica apical, lesão hipoxímica) ${ }^{2,21}$, o que, a nosso ver, indica fases diferentes da sua evoluçăo e sua interrelaçāo com a qualidade do músculo cardíaco adjacente. A lesāo, quando localizada na ponta do ventriculo esquerdo, é considerada, por muitos autores, como característica da doença de Chagas, devido à sua elevada freqüencia em necropsias (45\% a $86,1 \%)^{5,11,14,17,20}$.

A lesāo consiste, essencialmente, em um adelgaçamento da musculatura com justaposição do epicárdio ao endocárdio na regiāo afetada, mais comumente a ponta do ventriculo esquerdo, podendo acometer outras regiōes do ventrículo esquerdo, ou mesmo o ventrículo direito. $\mathrm{O}$ adelgaçamento resulta em um ponto fraco na parede do ventrículo esquerdo, que forma um abaulamento sistólico, em muitos casos verdadeiros aneurismas que apresentam formas diversas(dedo de luva, mamilo, raquete e sacular), transparentes à luz, e distorcendo a musculatura ventricular, com conseqüências para a contraçăo.

Segundo CARVALHAL et alii ${ }^{11}$, do ponto de vista histológico, a lesão é variável, constituindo-se de tecido colágeno proliferado e restos de processos necrobióticos. Existe um infiltrado, em graus variáveis, constituído de linfócitos e plasmócitos, podendo encontrar-se sangue.

A patogenia da lesão é bastante controvertida. Há muitas hipóteses, todas incapazes de elucidar os mecanismos de formaçāo da lesão.

Vários autores têm discutido o assunto, defendendo ou contraditando a participaçāo de mecanismos neurogênicos, hipoxêmicos, inflamatórios e mecânicos, sem, entretanto, econtrarem uma explicação convincente, seja com uma hipótese isolada ou através um conjunto envolvendo vários mecanismos $5,7,10,13,25$.

A multiplicidade de termos para definir a lesão está associada às fases da sua evoluçāo e a um fator importante, que é a funçāo ventricular esquerda. Quanto melhor o músculo, mais rápida será a capacidade de dilatação e a caracterização da lesão como aneurisma.

Parece bastante lógico que existam dois grupos de pacientes que devem ser separados. O primeiro, a grande maioria, é aquele com lesāo apical, bem ou mal definida, o músculo cardíaco severamente comprometido, com insuficiência cardíaca manifesta ou nāo. O seu prognóstico é sombrio, determinado pela má funçāo ventricular esquerda, pouco contribuindo o aneurisma para o estado clínico do paciente. O segundo grupo é o de pacientes com lesōes bem 
WANDERLEY NETO, J.; TORRES, L. D. F.; ELIAS, D. O.; SILVA, S. T.; GAMA, H. C.; FARIAS, M.; DOURADO, G. O.; CAVALCANTE, C. C.; WISZOMIRSKY, A. D. B.; MENDONÇA, J. T. - Aneurisma chagásico de ventrículo esquerdo: aspectos cirúrgicos em 29 casos. Rev. Bras. Cir. Cardiovasc., 7(3): 180-185, 1992.

definidas e músculo cardíaco normal ou pouco alterado. Neste grupo, oaneurisma pode serfator determinante do prognóstico e tem interesse dos pontos de vista clínico e cirúrgico. Seriam pacientes próximos à chamada forma intermediária da doença de Chagas. RASO ${ }^{22}$ encontrou a lesāo de ponta à necropsia em $65 \%$ dos casos, sendo que $36 \%$ apresentavam trombose apical e $4 \%$ tinham área cardíaca normal.

$\mathrm{Na}$ nossa casuística, a maioria dos pacientes apresentava área cardíaca e função ventricular esquerda normais ou próximas do normal.

A correlação entre morte súbita, aneurisma apical e arritmia foi demonstrada por MARINS et alii ${ }^{17}$.

Os aneurismas chagásicos costumam ser sede de trombos e focos de embolização sistêmica e pulmonar, confirmados por vários autores ${ }^{4,5,11,18,24,26}$.

A participação do aneurisma na gênese da insuficiência cardíaca é questionada ${ }^{23}$. Nos casos de pequenos aneurismas, acreditamos que pouco contribuem para o quadro, sendo a causa preponderante a falência do músculo cardíaco. Quando o aneurisma é do tipo sacular, como na Figura 2, não resta dúvida de que é a causa determinante da insuficiência cardíaca.

A rotura do aneurisma é uma ocorrência rara na literatura ${ }^{19}$.

Embora se tenha tentado estabelecer um padrão eletrocardiográfico para o aneurisma chagásico, hoje há consenso de que não existe um aspecto que defina a lesão; no entanto, raramente o eletrocardiograma é normal ${ }^{21.28}$.

Apesar de ser uma lesão bastante conhecida e freqüente, pouco sabemos sobre sua história natural.

ALBANESI FILHO \& GOMES FILHO ${ }^{3}$ publicaram uma série de 55 pacientes, com uma perda de seguimento de $35 \%$ e onde mesclam-se pacientes com e sem disfunçāo do músculo cardíaco. Torna-se necessária a caracterizaçăo de subgrupos para que possamos falar a mesma linguagem e encontrar respostas conclusivas.

A possibilidade de benefício pela cirurgia foi aventada por RIBEIRO ${ }^{23}$, em 1971, e, recentemente, MARINS et alii ${ }^{17}$ e CARRASCO et alii ${ }^{9} \mathrm{consideram} \mathrm{a}$ cirurgia uma opçāo terapêutica.

Somente em 1975, TEIXEIRA et alii ${ }^{27}$ ressecaram o primeiro aneurisma chagásico de paciente com tromboembolismo cerebral recorrente. Logo a seguir, ANDRADE et alii ${ }^{6} \mathrm{e}$ LAMOUNIER et alii ${ }^{16}$ publicaram casos operados com sucesso, para tratamento de taquiarritmias. Mais recentemente, 1986, BLANDÓN et alii ${ }^{8}$ relataram caso de paciente com cardiopatia chagásica crônica operado para tratar arritmia refratária a medicamento.
Na nossa casuística, 8 pacientes apresentaram embolia cerebral prévia; 6 deles com pequenos aneurismas de aspecto em dedo de luva ou mamilo, com colo estreito e músculo normal, sugerindo o foco da embolia. Em 2 pacientes, nos quais não havia evidência angiográfica de trombos, verificamos a presença deles de formação recente, indicando, como é admitido por outros autores ${ }^{5,26}$, uma grande incidência de tromboembolismo sistêmico e pulmonar, nāo diagnosticado em vida.

Um achado interessante e pouco encontrado foi - de duplo aneurisma e casos de aneurisma de parede inferior isolado, inclusive com insuficiência mitral grave, povavelmente por distorção do aparelho subvalvar mitral (Figura 4). Cabe, nesses casos, o diagnóstico diferencial com o aneurisma subvalvar idiopático, também chamado de aneurisma africano. Nessa lesão, a comunicação com a cavidade ventricular se faz através de múltiplos orifícios, os pacientes não são chagásicos, apresentam áreas calcificadas e aderentes ao pericárdio. A microscopia é diferente; há herniação do endocárdio, depósito de cálcio e nāo se identifica reaçāo inflamatória ${ }^{\text {. }}$

$\mathrm{O}$ aspecto angiográfico dos diversos tipos comparados seqüencialmente (Figura 2 ) sugere a dilataçăo progressiva da zona adelgaçada e do colo do aneurisma, até formar dilataçōes saculares que roubam parte do débito sistólico e distorcem o ventrículo esquerdo, podendo causar insuficiência cardíaca. No caso de localizaçăo em parede inferior, pode ocorrer insuficiência mitral.

Parece ser consensual que as lesōes aneurismáticas do ventrículo esquerdo com tromboelismo recidivante e arritmia incontrolável $6,16,27$ têm indicação cirúrgica indiscutível. Ressalve-se que, para maior segurança e garantia da eficácia da cirurgia, é necessário o mapeamento da cavidade ventricular para localização precisa do foco arritmogênico e, assim, evitar insucesso, como ocorreu em um de nossos casos.

Em casos de insuficiência cardíaca com aneurismas saculares, a operação parece bem indicada.

Os conceitos atuais para indicação cirúrgica no aneurisma chagásico fundamentam-se nos estabelecidos para os aneurismas isquêmicos: arritmias, tromboembolismo e insuficiência cardíaca.

A etiopatogenia e o aspecto morfológico do aneurisma chagásico são diferentes, indicando que devem ser revistos. Assim, grandes aneurismas saculares, de ponta ou de parede inferior, devem ser removidos, mesmo na ausência de insuficiência cardíaca, arritmias ou tromboembolismo. 
WANDERLEY NETO, J.; TORRES, L. D. F.; ELIAS, D. O.; SILVA, S. T.; GAMA, H. C.; FARIAS, M.; DOURADO, G. O.; CAVALCANTE, C. C.; WISZOMIRSKY, A. D. B.; MENDONÇA, J. T. - Aneurisma chagásico de ventrículo esquerdo: aspectos cirúrgicos em 29 casos. Rev. Bras. Cir. Cardiovasc., 7(3): 180-185, 1992.

Quanto aos pacientes com pequenos aneurismas, músculo normal e assintomáticos, não temos resposta ainda, quanto aos benefícios de uma cirurgia profilática e não existem informaçōes na literatura a respeito da evolução dos pacientes. Tornamse necessários metodologia apropriada com grupos uniformes e seguimento adequado, para definirmos morbidade e mortalidade correlacionadas à lesão, indicando-nos os reais benefícios do tratamento cirúrgico.

Concluímos que os aneurismas chagásicos podem ser ressecados cirurgicamente, com baixa mortalidade. A cirurgia pode aliviar os sintomas de pacientes com insuficiência cardíaca congestiva, tratar arritmias recidivantes e prevenir episódios tromboembólicos em pacientes selecionados.

WANDERLEY NETO, J.; TORRES, L. D. F.; ELIAS, D. O.; SILVA, S. T.; GAMA, H. C.; FARIAS, M.; DOURADO, G. O.; CAVALCANTE, C. C.; WISZOMIRSKY, A. D. B.; MENDONÇA, J. T. - Left ventricular aneurysm in Chagas' disease: surgical aspects in 29 cases. Rev. Bras. Cir. Cardiovasc., 7(3):180-185, 1992.

ABSTRACT: During the period between March 1980 and December 1991, 29 patients have been operated upon for removal of left ventricular aneurysms of Chagas' disease ethiology. There were 22 females and 7 males, with ages at operation ranging from 21 to 69 years. All of them had a positive immunologic reaction to Chagas disease; 8 patients presented preoperatively stroke, 8 patients had tiredness associated with heart failure, 8 patients had palpitations and seizures; 5 others presented multiple symptoms. Three patients had artificial pacemakers implanted. The electrocardiogram was abnormal in all cases; half of them had normal chest roentgenograms. Coronary arteriography was normal in all patients. Left ventricular angiogram demonstrated an apical lesion like a "nipple" in 14 cases, a saccular aneurysm in 8 cases, an inferior wall aneurysm in 4 cases, and combined apical and inferior aneurysms in 3 cases. One of the cases of double aneurysms had also severe mitral insufficiency. In 4 patients thrombi were detected in the left ventricular angiogram. There was no hospital mortality. During follow-up we registered a single death and 3 patients progressed to a complete heart block which required pacemaker insertion. Another patient had recurrence of preoperative arrhithmia. We conclude that surgical removal of left ventricular aneurysm associated with Chagas' disease in selected patients is a low mortality procedure, may prevent recurrent thromboembolysm, controls heart failure and may suppress arrhithmias in a significant number of patients.

DESCRIPTORS: Chagas' disease aneurysms, surgery; aneurysms, left ventricle, surgery.

\section{REFERÊNCIAS BIBLIOGRÁFICAS}

1 ABRAHAMS, D. G.; BARTON, C. J.; COCKSHOTT, W. P.; EDIGTON, G. M.; WEAVER, E. J. M. - Annular subvalvar left ventricular aneurysms. Q.J. Med., 31: 345-360, 1962.

2 ALBANESI FILHO, F. M. - Aneurismas mamilares apical e inferior na cardiopatia chagásica crônica. Arq. Bras. Cardiol., 56: 147-149, 1991.

3 ALBANESI FILHO, F. M. \& GOMES FILHO, J. B. M. - A lesão apical do ventrículo esquerdo na evoluçāo clínica da cardiopatia chagásica crônica. Arq. Bras. Cardiol., 56: 457-463, 1991. tromboembolismo em pacientes com lesảo apical da

cardiopatia chagásica crônica. Rev. Port. Cardiol., 10: 35-42, 1991.

5
ANDRADE, J. A. - A lesão apical do coraçāo na miocardite crônica chagásica. Hospital, 50: 59-72, 1956.

6 ANDRADE, J. C. S.; FORTE, V.; BUFFOLO, E. Taquiarritmias no paciente chagásico: diagnóstico etiológico e tratamento cirúrgico. Rev. Bras. Med., 33: 129-133, 1976.

BESTETTI, R. B.; FINZI, L. A.; OLIVEIRA, J. S. M. Chronic Chagas'heartdisease presenting as an impending myocardial infarction: a case favoring the neurogenic pathogenesis concept. Clin. Cardiol.,10: 368-370, 1987.

BLANDÓN, R.; LEÓN, L. E.; GONZALES, B. - Tratamiento 
WANDERLEY NETO, J.; TORRES, L. D. F.; ELIAS, D. O.; SILVA, S. T.; GAMA, H. C.; FARIAS, M.; DOURADO, G. O.; CAVALCANTE, C. C.; WISZOMIRSKY, A. D. B.; MENDONÇA, J. T. - Aneurisma chagásico de ventrículo esquerdo: aspectos cirúrgicos em 29 casos. Rev. Bras. Cir. Cardiovasc., 7(3): 180-185, 1992.

quirurgico de la arritmia cardiaca refractaria a medicamentos en paciente con cardiopatia chagasica cronica. Rev. Med. Panamá, 11: 164-170, 1986.

CARRASCO, H. A.; BARBOSA, J. S.; INGLESSIS, G.; FUENMAYOR, A.; MOLINA, C. - Left ventricular cineangiography in Chagas' disease: detection of early myocardial damage. Am. Heart J., 104: 595-609, 1982.

CARVALHAL, S. - A lesāo apical na cardiopatia chagásica. Arq. Bras. Cardiol., 25:257-262, 1972.

11 CARVALHAL, S.;PORTUGAL, O.; PALADINO, N.; YOUNES, A.; CAMPOS, C. M.; RAMOS, O.; UVO, D.; GEBARA, M. - Alteraçōes do complexo QRS nas derivaçōes precordiais e seu substrato anatômico em pacientes portadores de miocardite chagásica crônica. Rev. Paul. Med., 45: 29-36, 1954.

CHAGAS, C. - Processos patogênicos da tripanosomiase americana. Mem. Inst. Osvaldo Cruz, 8: 5-35, 1916.

13 FUENMAYOR, A. J.; RODRIGUES, L.; TORRES, A.; DONIS, J.; NAVAS, M.; FUENMAYOR, A. M.; DAVILA, D. - Valsalva maneuver: a test of the functional state of cardiac innervation in chagasic myocarditis. Int. J. Cardiol., 18: 351-355, 1988.

14 GRANZOTTI, J. A.; MARIN, N. J. A.; GALLO, Jr., L. Contribuição ao estudo do "aneurisma" da ponta na cardiopatia chagásica crônica. Arq. Bras. Cardiol.. 27: 477-482, 1974.

15 JATENE, A. D. - Left ventricular aneurysmectomy: resection or reconstruction. J. Thorac. Cardiovasc. Surg., 89: 321-331, 1985.

16 LAMOUNIER, E. N.; HERMAN, J. L. Y.; MARTINEZ, F. E. - Aneurismectomia como tratamento de taquiarritmia refratária em pacientes portadores de aneurisma ventricular de etiologia chagásica. Arq. Bras.Cardiol,,28: 549-556, 1975.

17 MARINS, N.; MIZIARA, H. L.; SOBRAL NETO, J.; MATOS, J. V.; DUMARESQ, L. C.; SOUZA, R. Taquiarritmias paroxísticas em chagásicos com lesão daponta: correlaçāoanátomo-eletrocardiográfica. Folha Med. (Brasil), 83: 175-178, 1981.

18 OLIVEIRA, J. S. M.; ARAÚJO, R. L. C.; NAVARRO, M. A.; MUCCILLO, G. - Cardiac thrombosis and throm-

boembolism in chronic Chagas' heart disease. Am. J. Cardiol., 52: 147-151, 1983.

OLIVEIRA Jr., W. A.; SALAZAR, L. F.; CALADO, I.; ALBUQUERQUE, M. A.; GARRET Jr., M.; CANTARELLI, E. L.; ASSI, N. - A lesāo apical na miocardiopatia chagásica. Ars Curandi Cardiol., 7: 30-42, 1985.

RASO, P. - Contribuição ao estudo da lesão vorticilar na cardite chagásica crônica. Belo Horizonte, 1964. [Tese. Doutorado. Faculdade de Medicina de Belo Horizonte].

23 RIBEIRO, J. P. A. - A importância da área de acinesia apical na contratilidade do coraçāo chagásico crônico: perspectiva cirúrgica. Arq. Bras. Cardiol., 24: 31-36, 1971.

ROMANO, D.; SILVA, A. Q.; CUNHA, G. P.; OLIVEIRA, P. F. - Tromboembolismo na doença de Chagas. Rev. Med. Paraná, 25: 263-276, 1956.

ROSSI, M. A.; SANTOS, R. R.; GONÇALVES, S. Pathogenesis of the apical aneurysm in experimental Trypanosoma cruzi cardiomyopathy in BALC/mice. Fransac. Royal Soc. Tropic. Med. Hygiene, 80: 990$991,1985$.

SOBRAL NETO, J.; DUARTE, L.; BRESANI, J.; MATOS, J.; MIZIARA, H.; MARINS, N. - Tromboembolismo na cardiopatia chagásica crônica: estudo anatomopatológico. Arq. Bras. Cardiol., 30: 201-203, 1977.

27 TEIXEIRA, J.; BARROSO, M.; OLIVEIRA, P. S. - Aspectos cirúrgicos do aneurisma chagásico do ventrículo esquerdo: a propósito de um caso operado. Rev. Bras. Med., 32: 221-227, 1975.

28 VELOSO, C.; SALGADO, J. A.; ELIAN, A. A. - Estudo da lesão da ponta em relação ao padrāo de zona eletricamente inativável na cardiopatia chagásica crônica: correlaçāo anatomo-eletrocardiográfica. Arq. Bras. Cardiol., 17: 505-518, 1964. 